\title{
A new endoscopic therapy for an intraluminal diverticulum of the duodenum
}

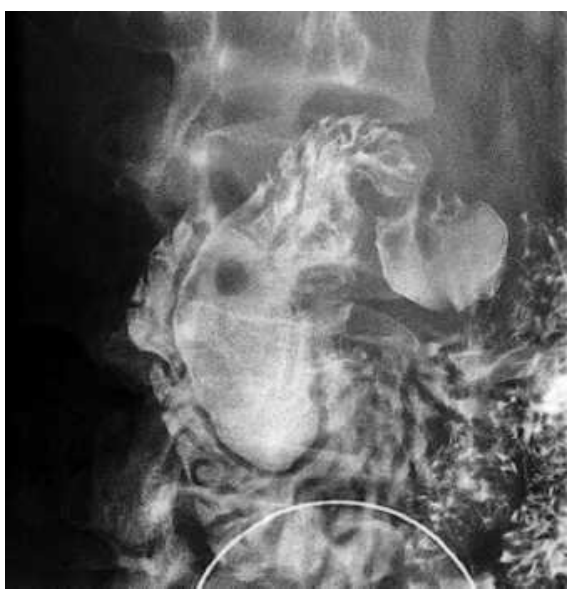

Figure 1 Conventional radiograph showing intraluminal diverticulum of the duodenum projecting within the lumen of the second duodenum.

We report the case of a 33-year-old woman with dyspeptic symptoms and $5 \mathrm{~kg}$ weight loss in 5 months.

During upper gastrointestinal endoscopy at another institution, access to the second part of the duodenum had not been possible for reasons that were unclear. At our hospital, a barium follow through revealed a sac-like contrast-containing structure within the duodenal lumen, suggestive of an intraluminal diverticulum of the duodenum (IDD) (Figure $\mathbf{1}$ and 2). Repeat endoscopy confirmed that the second portion of the duodenum could only be reached through a tiny passage at the level of the genu superius, laterally to the diverticular orifice.

After obtaining consent and with the aid of a therapeutic endoscope, we punctured the bottom of the diverticulum with a needle knife papillotome. A 0.035-inch guide wire was introduced in the hole, and hydrosoluble contrast material was injected above and below the puncture site to confirm the exact localization of the IDD (Figure 3). Consequently the puncture site was enlarged using a 7-Fr cystogastrotome, allowing the introduction of an insulated-tip needle knife to incise the diverticular wall longitudinally,

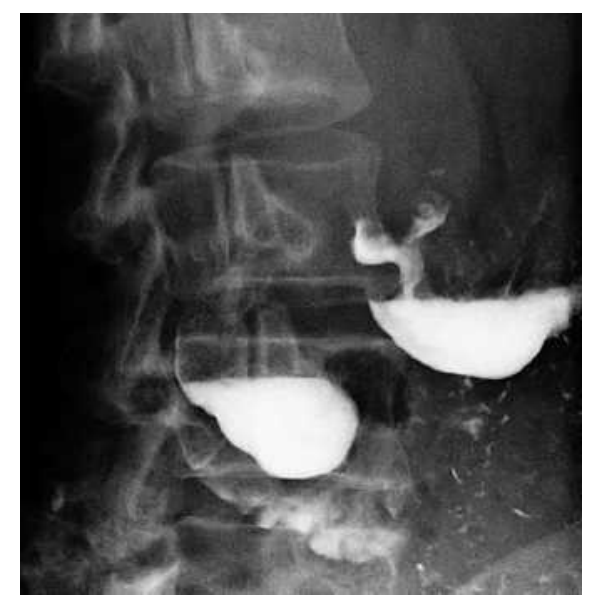

Figure 2 Barium retention in both the bulbus and intraluminal diverticulum of the duodenum.

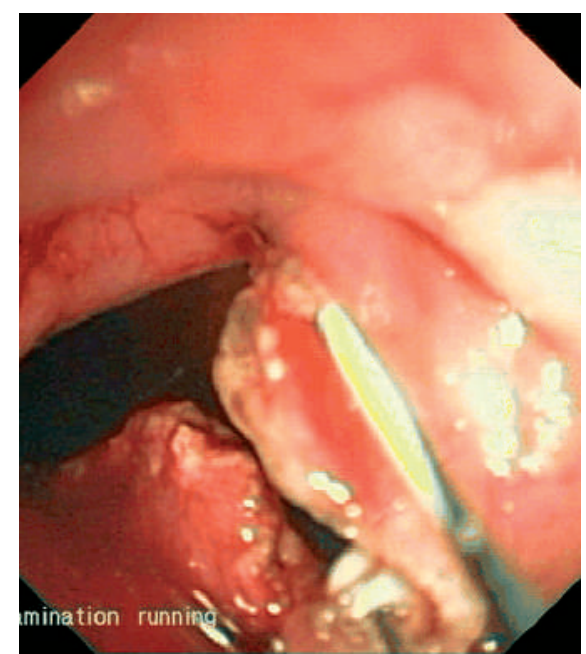

Figure 4 Endoscopic view from within the diverticulum, showing progression of the diverticulotomy using the insulated-tip knife; note the presence of the guide wire in the incision orifice.

\section{Videos 1-3}

Sequence 1 Inspection of the intraluminal diverticulum of the duodenum. Sequence $\mathbf{2}$ Perforation of the diverticular sac using a needle-knife papillotome. Sequence 3 Progressive incision of the diverticular septum with the insulated-tip knife.

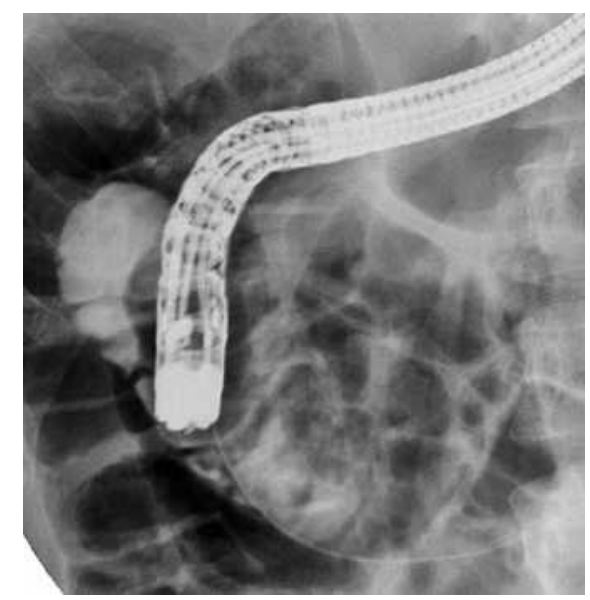

Figure 3 Hydrosoluble contrast injection in the diverticulum and in the duodenal lumen through the perforation of the diverticulum.

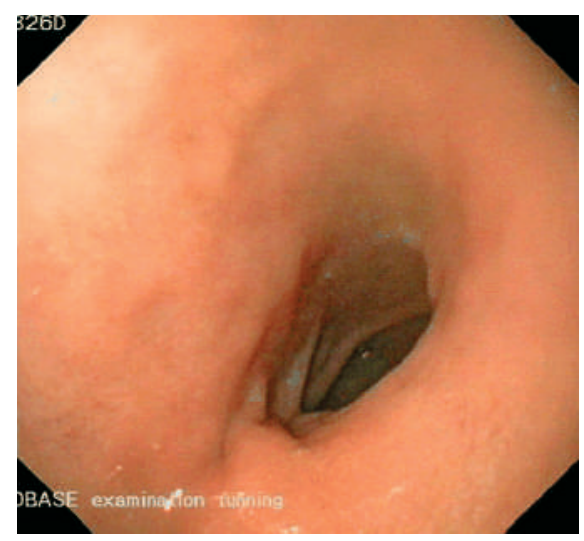

Figure 5 Endoscopic view of the genu superius from the bulbus showing complete healing of the mucosa and patency of the duodenum.

up to the diverticular mouth at the genu superius (Figure 4 and video sequences). Endoscopic control with side-viewing scope identified both the papilla and the presumed location of the intramural part of the common bile duct laterally to the section. No immediate or delayed complication occurred. A control endoscopy was performed 1 month later showing complete healing and easy passage to the second duodenum (Figure 5). Clinical evaluation, 8 months postprocedure revealed continued absence of the dyspeptic symptoms, and no additional weight loss.

DOI: $10.1055 / s-2006-945175$ 


\section{UCTN}

IDD is a rare developmental anomaly found in adulthood [1-3]. The main symptoms are related to partial or intermittent obstruction. The most common complications include bleeding, acute or recurrent pancreatitis, cholangitis, and intussusception [3,4]. Several techniques of endoscopic management of IDD have been described $[4,5]$. The present procedure allows avoidance of trauma to the papilla, the common bile duct, or the opposite duodenal wall.

Endoscopy_UCTN_Code_TTT_1AO_2AN

\section{A. Badaoui, H. Piessevaux}

Department of Gastroenterology, Cliniques universitaires St-Luc, Brussels, Belgium.
References

${ }^{1}$ De Castro ML, Hermo JA, Pineda JR et al. Acute bleeding and anemia associated with intraluminal duodenal diverticulum: case report and review. Gastrointest Endosc 2003; 57: 976 - 979

2 Ravi J, Joson PM, Ashok PS. Endoscopic incision of intraluminal duodenal diverticulum. Case report of a new technique. Dig Dis Sci 1993; 38: $762-766$

${ }^{3}$ Finnie IA, Ghosh P, Garvey $C$ et al. Intraluminal duodenal diverticulum causing recurrent pancreatitis: treatment by endoscopic incision. Gut 1994; 35: 557 - 559

${ }^{4}$ Lee SH, Park SH, Lee JH et al. Endoscopic diverticulotomy with an isolated-tip papillotome (Iso-Tome) in a patient with intraluminal duodenal diverticulum. Gastrointest Endosc 2005; 62: 817-819

${ }^{5}$ Hiraoka T, Nakamura M, Ohno K et al. Endoscopic excision of intraluminal duodenal diverticulum. Dig Dis Sci 1985; 30: 274-281
Corresponding author

\section{H. Piessevaux, MD}

Department of Gastroenterology Cliniques universitaires St-Luc Université catholique de Louvain Avenue Hippocrate 10

1200 Brussels

Belgium

Fax: +32-2-764-8927

Email: piessevaux@gaen.ucl.ac.be 